\title{
ON THE CONSIDERATION OF UNCERTAINTY IN DESIGN: OPTIMIZATION - RELIABILITY - ROBUSTNESS
}

\author{
N. Lelièvre ${ }^{1,3}$, P. Beaurepaire ${ }^{2,3}$, C. Mattrand ${ }^{2,3}$, N. Gayton ${ }^{2,3}$, A. Otsmane ${ }^{4}$ \\ ${ }^{1}$ Université Clermont Auvergne, Institut Pascal \\ BP 10448, F-63000 CLERMONT-FERRAND \\ e-mail: nicolas.lelievre@sigma-clermont.fr \\ ${ }^{2}$ Université Clermont Auvergne, SIGMA Clermont, Institut Pascal \\ BP 10448, F-63171 AUBIERE \\ e-mail: \{pierre.beaurepaire, cecile.mattrand, nicolas.gayton \}@ sigma-clermont.fr \\ ${ }^{3}$ CNRS, UMR 6602, Institut Pascal \\ F-63171 AUBIERE \\ ${ }^{4}$ SNECMA Site Villaroche \\ Rond-Point René Ravaud 77550 MOISSY-CRAMAYEL CEDEX \\ e-mail: abdelkader.otsmane@ snecma.fr
}

Keywords: Optimization, Design, Robustness, Reliability

\begin{abstract}
Structural designs aspire to ensure the functionality of a system respecting specific requirements defined by engineers. Structural optimization techniques are widely used to optimize the system performances while garanteeing that specific requirements are fulfilled. However, it is worth remembering that uncertainties might affect all design quantities which can make the design problem much more arduous to solve. This paper aims to discuss the formulation of design problems under uncertainty. Several strategies might be identified when considering uncertainties in the objective and/or constraint functions. Moreover, this paper aspires to clarify the notions of robustness and reliability. After a presentation of each formulation, an academic example illustrates the different strategies and shows the main differences between results of each one. Thus, the choice of the formulation should be viewed as a crucial step of design procedure.
\end{abstract}




\section{Introduction}

During the design of a structure, engineers can aim to two main strategies. On one hand, structures have to be able to resist the environmental loads. The structure is thus characterized by an analytical or numerical model which can be used to identify the admissible designs (see e.g. [1]). Designer can adapt the structure parameters to prevent failures and identify an appropriate design. The second strategy is the optimization of the structure which consists of maximize (resp. minimize) the performance (resp. cost) of the structure. The two strategies can obviously be coupled to constrained optimization which provides the best admissible design.

Parameters from structural design problems are in practice affected by uncertainties which may be caused by a lack of knowledge of the production condition for instance or by intrinsic uncertainties. To take into account these uncertainties in design, engineers often use simplifying hypothesis such as safety factors or considering only a reference value. Nevertheless, the variability of the uncertain parameters cannot be considered completely by these methods which can be perform accounting explicitly for uncertainties with probabilities or imprecise probabilities.

In the literature, many papers on design under uncertainty are relating to numerical methods to solve these problem (see e.g. [2]) whereas the objective of the present paper is the clarification of problem formulations. Indeed, stochastic formulations of constraint and objective functions are described. Two main notions are explained: robustness which is habitually associated with the consideration of uncertainties in objective functions and reliability which is based on the introduction in the constraint functions. The proposed classification presented in Table 1 is based on an overview of the literature on the topic (see e.g. [3, 4]).

The paper is structured as follows: section 2 discusses the general concept of uncertainties and also proposes our classification of design formulations. Based on this classification, section 3 presents three deterministic design formulations. Section 4 introduces five formulations for design under uncertainty. An academic example then compares the results obtained from each formulation in section 5. Finally, some conclusions are drawn in section 6 .

\section{Design under uncertainty}

\subsection{Sources of uncertainty and their classification}

Engineers face uncertainties during the design process, which might affect any parameter. Two catergories are commonly identify in the structural engineering community, namely aleatory or random uncertainties and epistemic uncertainties, see e.g. [5, 6]. On one hand, aleatory or random uncertainties refer to those that cannot be reduced by introducing additional data or improving the modeling process. They therefore should be viewed as inherent or intrinsic to the considered phenomenon and, as Beyer and Sendhoff [5] pointed out, "the designer has to "live with them" and optimize his design according to this reality". On the other hand, epistemic uncertainties are only due to a lack of knowledge of the studied phenomenon or system behavior, and could therefore be reduced if some conceivable efforts are undertaken.

In a design framework, another classification for uncertainties might be proposed, as suggested by several authors [5,7]. From their standpoint, uncertainties can be distinguished depending on whether they can be acted upon or not in the design phase:

- Type I uncertainties are primitively linked to the environment and conditions of use. The variables that show this type of uncertainty are hereafter noted in the series $P_{j}(\omega), j=$ $1, \ldots, m$ and stored in vector $\mathrm{P}(\omega)$. They do not play a role in the design procedure, i.e. 
they are independent from it, and as such they are not design variables.

- System function uncertainties are those linked to the evaluation of the performance (or output) of the system. In this paper, these uncertainties are also gathered in vector $\mathrm{P}(\omega)$.

- Feasibility uncertainties are associated with uncertainties on the constraint functionsand have hence an influence on the definition of the design space. These uncertainties are also considered as model uncertainties and grouped in vector $\mathrm{P}(\omega)$.

- Type II uncertainties are those connected with the production/ manufacturing process. Geometrical variables noted $X_{i}(\omega), i=1, \ldots, n$ (and stored in vector $\mathrm{X}(\omega)$ ) are usually linked to this type of uncertainty. They are part of the design variables which might affect the performance of the system.

\subsection{Consideration of uncertainties in engineering}

In the design process, uncertainties can be considered by two approaches. The first approach is the conversion of each uncertain parameter into a reference value. The stochastic problem thus becomes a deterministic problem. The so-called worst-case method permits to identify this reference value. Indeed, in this method, uncertain parameters are assumed to be bounded, and the bound of each variable associated with the worst scenario is identified. In tolerance analysis, this method is commonly used (see e.g. [8]) because each dimensions are uncertain and the tolerance intervals provide suitable bounds. However, this strategy is not applicable to general problems and the identification of the bounds may be a complex task. The second method to convert a random variable into a deterministic parameter consists of weighting each uncertain parameter by a safety factor commonly, which depends on the importance of the variable. With this method, unbounded variable can also be treated.

The second approach is the explicit consideration of uncertainties which are characterized by a mathematical representation. The variation of uncertain parameters can thus be taken entirely into account and it allows the propagation uncertainties to the response of the structure. Several approaches may be used to realize this characterization. The probabilistic approach defines each uncertain variable by a distribution function (see e.g. [9]) and it is commonly used in mechanical design (see e.g. [10]). An alternative approach is imprecise probabilities. The possibilistic approach defines uncertain parameters by their possibility distributions, as detailed e.g. by Zadeh [?]. Another method is using fuzzy sets to characterize uncertainties (see e.g. $[?])$.

\subsection{Design requirements}

In design processes, the state of mechanical system's performances can be characterized by two different functions. First, the objective-type functions $f(\mathrm{X}(\omega), \mathrm{P}(\omega))$ aim to quantify the performances of the mechanical system. Hence, optimization consist of maximizing the performances of the system (e.g. quality level), minimizing the cost or targeting a reference value. Other design-related functions are the constraint-type functions $g(\mathrm{X}(\omega), \mathrm{P}(\omega))$ which must be satisfied in all operating conditions to ensure the functionality of the system. These constraint functions must be achieved as closely as possible in order not to degrade the objective functions. The constraint functions are used to define the admissible space which is defined as $\left\{\mathrm{X} \in \mathbb{R}^{n} \mid g(\mathrm{X}(\omega), \mathrm{P}(\omega)) \geq 0\right\}$. 


\subsection{Proposed classification of design formulations}

During design process, uncertainties can be accouted by several strategies, which involves multiple design problem formulations. Concepts such as reliability and robustness need to be introduced. A classification is proposed by Gang [11] who brings forward five design problem formulations. Our classification is introduced in Table 1, where eight design formulations are considered making the distinction by taking into account uncertainties in the objective and/or the constraint functions. Robustness is defined by the faculty of a system's response to be insensitive to small variations in system parameters, and is thus associated with the objective function. When uncertainties are taken into consideration in the constraint functions, non-admissible solutions are tolerated as long as they remain rare. Reliability is therefore associated with the constraint functions; it characterizes the ability of a system to ensure its functions in a given context.

Three possible states can be defined for each output function: no function is used, a deterministic function is employed or a function subject to uncertainties is considered. Nine combinations are thus introduced according to the state of objective and constraint functions in Table 1 and are detailed in the next sections.

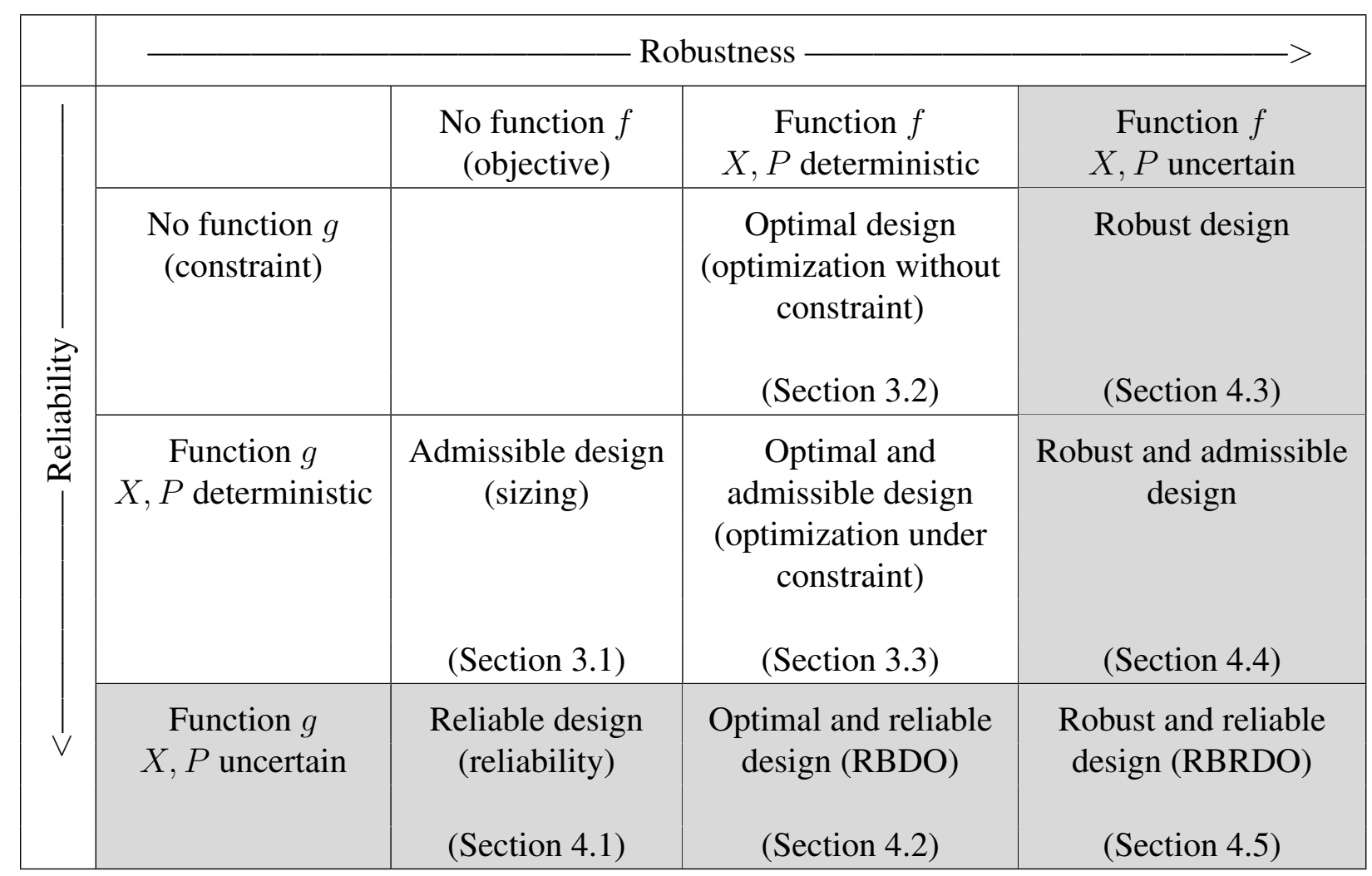

Table 1: Different design approaches. Grey boxes take into account some uncertainties

\section{Deterministic design formulation}

\subsection{Admissible design - Sizing}

Sizing is the most often used scheme in design because of it ease of implementation. Indeed, the admissible design corresponds to the set of solutions that respect problem constraints and thus provides the solution space of design variables. This design formulation (Equation (1)) is 
completely deterministic.

$$
\text { Find } \overline{\mathrm{X}}_{A d m} \text { such that : } g\left(\overline{\mathrm{X}}_{A d m}, \mathrm{P}^{(k)}\right) \geq 0
$$

However, dealing with uncertainties in an implicit manner can be realized by transforming the initial stochastic problem into a deterministic problem using the worst-case approach or the safety factors

\subsection{Optimal design - Optimization without constraint}

The principle of optimal design is to optimize an objective function which is not subject to constraints. This problem formulated in Equation (2) is entirely deterministic.

$$
\text { Find } \overline{\mathrm{X}}_{O p t} \text { such that : } \overline{\mathrm{X}}_{O p t}=\underset{\overline{\mathrm{X}}}{\operatorname{Arg} \max } f\left(\overline{\mathrm{X}}, \mathrm{P}^{(k)}\right)
$$

Industrial problems cannot be treated with this design formulation since this is an unconstrained optimization and can thus lead to non-admissible solutions (see section 3.1). However, this design formulation can be applied for mathematical applications such as for instance linear and non-linear regressions solved by the least-square method.

\subsection{Optimal and admissible design - Optimization under constraint}

The optimal and admissible design in the combination of the two previous formulations since using only one of the two formulations is generally insufficient for designers. Hence, the obtained design is both optimal with respect to the objective function and admissible with respect to the constraint functions (Equation (3)).

$$
\begin{aligned}
& \text { Find } \overline{\mathrm{X}}_{\text {OptAdm }} \text { such that : } \\
& \overline{\mathrm{X}}_{\text {OptAdm}}=\underset{\overline{\mathrm{X}}}{\operatorname{Arg} \max } f\left(\overline{\mathrm{X}}, \mathrm{P}^{(k)}\right) \\
& \text { Subject to (s.t.) } g\left(\overline{\mathrm{X}}, \mathrm{P}^{(k)}\right) \geq 0
\end{aligned}
$$

This is a classic problem of optimization under constraint without considering uncertainties, which is also called deterministic constrained optimization.

\section{Formulation of design in an uncertain context}

\subsection{Reliable design}

Reliable design formulation can be viewed as an extension of the admissible design problem introduced in section 3.1. In this approach, uncertainties are explicitly taken into account. They may be modeled by the probabilistic approach, as briefly recalled in section 2.2 , thereby leading to an assessment of the failure probability of a system or structure and guaranteeing that it is below a given threshold value $P_{\text {target }} . \overline{\mathrm{X}}$ denotes therefore the mean of the probability law of $\mathrm{X}$.

$$
\begin{aligned}
& \text { Find } \overline{\mathrm{X}}_{\text {Rel }} \text { such that: } \\
& \text { Prob }\left(g\left(\mathrm{X}\left(\overline{\mathrm{X}}_{\text {Rel }}, \omega\right), \mathrm{P}(\omega)\right) \leq 0\right) \leq P_{\text {target }}
\end{aligned}
$$

The failure probability threshold value may be fixed using a risk-based approach (e.g. [12]) for which consequences of failure are evaluated. In the case of major failure events $P_{\text {target }}$ needs to be very low, whereas its value can be higher if failure events have minor consequences. 


\subsection{Optimal and reliable design - Reliability-Based Design Optimization}

Optimal and reliable design formulation consists of optimizing a deterministic objective function subject to stochastic constraints. This design, commonly named reliability-based design optimization (RBDO), is formulated in Equation (5) as suggested by $[13,14,15]$. In this paper, RBDO is always associated with deterministic objective and probabilistic constraints.

$$
\begin{aligned}
& \text { Find } \overline{\mathrm{X}}_{\text {OptRel }} \text { such that: } \\
& \overline{\mathrm{X}}_{\text {OptRel }}=\underset{\overline{\mathrm{X}}}{\operatorname{Arg} \max } f\left(\overline{\mathrm{X}}, \mathrm{P}^{(k)}\right) \\
& \text { s.t. : } \operatorname{Prob}(g(\mathrm{X}(\overline{\mathrm{X}}, \omega), \mathrm{P}(\omega)) \leq 0) \leq P_{\text {target }}
\end{aligned}
$$

Reliability-based design optimization has been widely discussed in the literature and alternative formulations might be found where for instance uncertainties are considered in both objective and constraint functions [16, 17].

\subsection{Robust design}

Such as the optimal design, robust design formulation consists of optimizing an objective function without taken any constraints into account (Equation (6)). The main difference is that uncertainties are taken into account in the objective function $(\Psi)$ and which is thus named robust objective function.

$$
\begin{aligned}
& \text { Find } \overline{\mathrm{X}}_{R o b} \text { such that: } \\
& \overline{\mathrm{X}}_{R o b}=\underset{\overline{\mathrm{X}}}{\operatorname{Arg} \max } \Psi(\mathrm{X}(\overline{\mathrm{X}}, \omega), \mathrm{P}(\omega))
\end{aligned}
$$

The choice of formulation for the robust function is essential, since it can significantly modify the results of the optimization. Many formulations can be used for the robust function, from basic ones to more elaborate formulas such as linear combination ([18]), Taguchi's Mean Square Deviation $([5,19])$ or the Mean Square Error ([20]). This formulation is generally not relevant for industrial problem because of the lack of constraints.

\subsection{Robust and admissible design}

Robust and admissible design consists of optimizing the robust objective function $(\Psi)$ subject to deterministic constraint functions (Equation (7)).

$$
\begin{aligned}
& \text { Find } \overline{\mathrm{X}}_{\text {RobAdm }} \text { such that: } \\
& \overline{\mathrm{X}}_{\text {RobAdm }}=\underset{\overline{\mathrm{X}}}{\operatorname{Arg} \max } \Psi(\mathrm{X}(\overline{\mathrm{X}}, \omega), \mathrm{P}(\omega)) \\
& \text { s.t. : } g\left(\overline{\mathrm{X}}, \mathrm{P}^{(k)}\right) \geq 0
\end{aligned}
$$

In this formulation, uncertainties are considered only in the objective functions, the constraint functions remain deterministic. The obtained optimum performance is also insensitive with respect to moderate variations of the variables.

\subsection{Robust and reliable design}

The last formulation is the robust and reliable design where uncertainties are explicitly taken into account in both objective and constraint functions (Equation (8)). Solutions are thus both 
robust and reliable. This formulation is sometimes called reliability-based robust design optimization (RBRDO) ([20, 21]).

$$
\begin{aligned}
& \text { Find } \overline{\mathrm{X}}_{\text {RobRel }} \text { such that : } \\
& \overline{\mathrm{X}}_{\text {RobRel }}=\underset{\overline{\mathrm{X}}}{\operatorname{Arg} \max } \Psi(\mathrm{X}(\overline{\mathrm{X}}, \omega), \mathrm{P}(\omega)) \\
& \text { s.t. : } \operatorname{Prob}(g(\mathrm{X}(\overline{\mathrm{X}}, \omega), \mathrm{P}(\omega)) \leq 0) \leq P_{\text {target }}
\end{aligned}
$$

This formulation might be viewed as the most complete formulation which provides the surest solutions.

\section{Application example}

This section aims to illustrate each design formulation on an academic example. The application used to highlight the effect of design formulation on the solution is the study of a cylindrical container. This container is defined by a radius $R$ and a height $h$ and must be able to contain a minimum of $33 \mathrm{~cm}^{3}$ whilst using a minimum of material. The container is manufactured using metal sheet with the same thickness for each side. Hence, the constraint function is defined by Equation (9) and the objective function, which represents the area of material, is defined by Equation (10).

$$
\begin{gathered}
g(R, h)=V(R, h)-33=\pi R^{2} h-33 \\
f(R, h)=2 \pi R h+2 \pi R^{2}
\end{gathered}
$$

The constraint function must be positive to ensure the sufficient volume of the container. The objective function must be minimized to obtain the design minimizing the quantity of material. The search of each design is bounded to $1 \leq R \leq 4$ and $1 \leq h \leq 10(\mathrm{~cm})$ and results are given in Table 2.

\section{Deterministic design}

The non-admissible domain is defined by the constraint function (Equation (9)) and is represented by a hatched area in Figure 1. Without any constraint, the minimal quantity of material is zero, the optimal design is thus the zero solution.To best respect the constraint, the constraint function must be as close to the equality as possible. The optimal and admissible solution is thus at the limit between the admissible and non-admissible domains (see continuous curve Figure 1).

\section{Design in an uncertain context}

The variables $R$ and $h$ are modeled by Gaussian distributions with the same standard deviation of $0.5 \mathrm{~mm}$, independently of their nominal value. The failure probability if set at $P_{\text {target }}=0.1$. The reliable design domain is bounded by this limit and the non-reliable domain is represented by the hatched area in Figure 2.

For robust optimization, a new objective function must be defined considering uncertainties. Here, a linear combination (Equation (11)) of the expectation $(E(\circ))$ and the standard deviation $(\sigma(\circ))$ of the initial objective function is chosen.

$$
\Psi(R, h)=0.25 \times E(f(R, h))+0.75 \times \sigma(f(R, h))
$$


Since no constraints are taken into account, the robust design solution is the zero solution (as it happens for optimal design).

As discussed previously, to best respect constraints, the equality mus be targeted by the constraint function. The obtained RBDO solution, thus, appears at the limit between the reliable and non-reliable domains (see dotted curve in Figure 1) whereas the robust and admissible design (resp. robust and reliable design) is at the limit between the admissible and non-admissible domains (resp. reliable and non-reliable design). The main difference between each solution is that the robust solutions are more insensitive to variations in the problem parameters.

This application shows the main differences between the formulations, which therefore should be considered as an essential component of the design process in order to obtain the desired design.

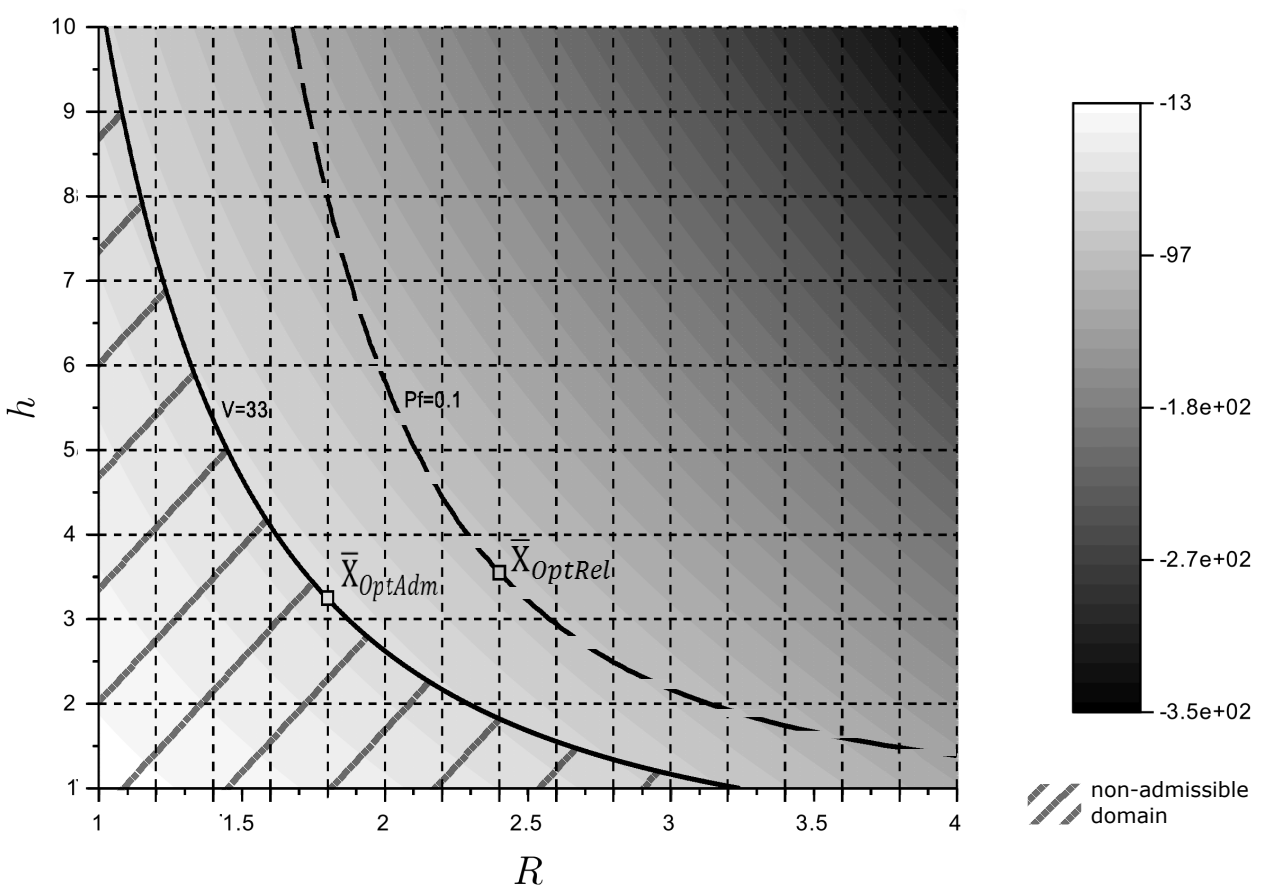

Figure 1: Example results - objective function $(-f(R, h)$ to be maximized) in grayscale 


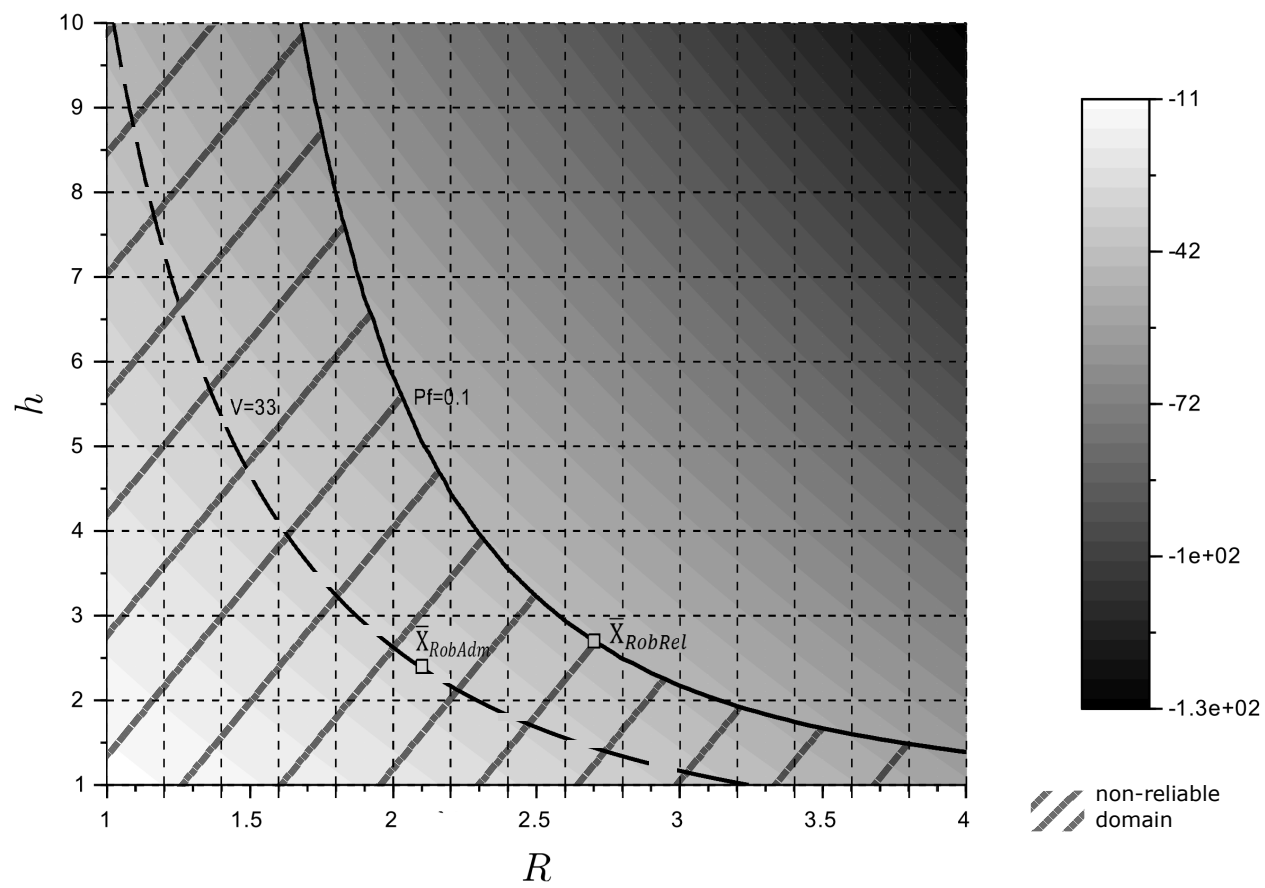

Figure 2: Example results - objective function $(-\Psi(R, h)$ to be maximized) in grayscale

\begin{tabular}{|c|c|c|c|c|}
\hline & & & stness & $\longrightarrow$ \\
\hline & & $\begin{array}{l}\text { No function } f \\
\text { (objective) }\end{array}$ & $\begin{array}{c}\text { Function } f \\
X, P \text { deterministic }\end{array}$ & $\begin{array}{c}\text { Function } f \\
X, P \text { uncertain }\end{array}$ \\
\hline$\stackrel{2}{:}$ & $\begin{array}{l}\text { No function } g \\
\text { (constraint) }\end{array}$ & & $\begin{array}{l}\text { Optimal design } \\
\overline{\mathrm{X}}_{O p t}=[0 ; 0]\end{array}$ & $\begin{array}{l}\text { Robust design } \\
\overline{\mathrm{X}}_{R o b}=[0 ; 0]\end{array}$ \\
\hline$\frac{.0}{20}$ & $\begin{array}{c}\text { Function } g \\
X, P \text { deterministic }\end{array}$ & $\begin{array}{c}\text { Admissible design } \\
\text { see Figure } 1\end{array}$ & $\begin{array}{c}\text { Optimal and } \\
\text { admissible design } \\
\overline{\mathrm{X}}_{\text {OptAdm }}=[1.8 ; 3.25]\end{array}$ & $\begin{array}{l}\text { Robust and admissible } \\
\text { design } \\
\overline{\mathrm{X}}_{\text {RobAdm }}=[2.1 ; 2.4]\end{array}$ \\
\hline$v$ & $\begin{array}{c}\text { Function } g \\
X, P \text { uncertain }\end{array}$ & $\begin{array}{c}\text { Reliable design } \\
\text { see Figure } 1\end{array}$ & $\begin{array}{l}\text { Optimal and reliable } \\
\text { design (RBDO) } \\
\overline{\mathrm{X}}_{\text {OptRel }}=[2.4 ; 3.55]\end{array}$ & $\begin{array}{l}\text { Robust and reliable } \\
\text { design (RBRDO) } \\
\overline{\mathrm{X}}_{\text {RobRel }}=[2.4 ; 2.7]\end{array}$ \\
\hline
\end{tabular}

Table 2: Summary of results for the container application.

\section{Conclusion}

In this paper, a comprehensive summary of design formulations is discussed by proposing a classification of design problems (Table 1). The different definitions and formulations are clarified. Designing aspires to identify admissible solutions subject to constraints, and optimizing 
aims to maximize performance metrics. Robustness is linked to the objective whereas reliability is associated with the constraints. The application shows that significant differences exist between each formulations. The choice of design formulation is thus a crucial step in the design procedure, and requires careful consideration.

\section{acknowledgements}

The first author was funded by a ministerial grant from Blaise Pascal University. The contribution of SNECMA Villaroche is also gratefully acknowledged.

\section{REFERENCES}

[1] H. Al Nageim, F. Durka, W. Morgan, D.T. Williams, Structural mechanics: loads, analysis, materials and design of structural elements, 7th edition, Pearson Education, London, 2010.

[2] M.A. Valdebenito, G.I. Schuëller, A survey on approaches for reliability-based optimization, Structural and Multidisciplinary Optimization, 42(5), 1-19, 2010a.

[3] I. Doltsinis, Z. Kan, Robust design of structures using optimization methods, Computer Methods in Applied Mechanics and Engineering, 193(23-26), 2221-2237, 2004.

[4] H.A. Jensen, M.A. Valdebenito, G.I. Schuëller, Kusanovic D.S. , Reliability-based optimization of stochastic systems using line search, Computer Methods in Applied Mechanics and Engineering, 198(49-52),3915-3924, 2009.

[5] H.G. Beyer, B. Sendhoff, Robust optimization - A comprehensive survey, Computer Methods in Applied Mechanics and Engineering, 196, 3190-3218, 2007.

[6] A. Der Kiureghian, O. Ditlevsen, Aleatory or epistemic ? Does it matter ?, Structural Safety, 31, 105-112, 2009.

[7] W. Chen, J.K. Allen, K.L. Tsui, F. Mistree, A Procedure for Robust Design: Minimizing Variations Caused by Noise Factors and Control Factors, ASME Journal of Mechanical Design, 118, n 4, 478-485, 1996.

[8] W.H. Greenwood, K.W. Chase, A new tolerance analysis method for designers and manufacturers, Transaction of the ASME, 109, 112-116, 1987.

[9] A.S. Nowak, K.R. Collins, Reliability of Structures, McGraw-Hill Higher Education, 2000.

[10] Z. Meng, G. Li, B.P. Wang, P. Hao, A hybrid chaos control approach of the performance measure functions for reliability-based design optimization, Computers and Structures, 146, 32-43, 2015.

[11] W. Gang, S. Wang, F. Xiao, D. Gao, Robust optimal design of building cooling systems considering cooling load uncertainty and equipment reliability, Applied Energy, 159, 265$275,2015$.

[12] R. Rackwitz, Optimization and risk acceptability based on the Life Quality Index, Structural Safety, 24, 397-331, 2002. 
[13] Z. Meng, P. Hao, G. Li, B. Wang, K. Zhang, Non-probabilistic reliability-based design optimization of stiffened shells under buckling constraint, Thin-Walled Structures, 94, 325$333,2015$.

[14] Z.P. Mourelatos, J. Liang, A Methodology for Trading-Off Performance and Robustness Under Uncertainty, Journal of Mechanical Design, 128, 856-863, 2006.

[15] E.H. Vanmarcke, Matrix formulation of reliability analysis and reliability-based design, Computers and Structures, 3, 757-770, 1973.

[16] I. Enevoldsen, J.D. Sørensen, Reliability-based optimization in structural engineering, Structural Safety, 15, 169-196, 1994.

[17] G.I. Schuëller, H.A. Jensen, Computational methods in optimization considering uncertainties -- An overview, Computer methods in applied mechanics and engineering, 198, $2-13,2008$

[18] M. Papadrakakis, N.D. Lagaros, V. Plevris, Design optimization of steel structures considering uncertainties, Engineering Structure, 27, no. 9, 1408-1418, 2005.

[19] M.W. Trosset, Taguchi and Robust Optimization, 1997.

[20] A.F. Shahraki, R. Noorossama, Reliability-based robust design optimization: A general methodology using genetic algorithm, Computers and Industrial Engineering, 74, 199207, 2014.

[21] I. Lee, K.K. Choi, L. Du, D. Gorsich, Dimension reduction method for reliability-based robust design optimization, Computers and Structures, 86, 1550-1562, 2008. 